\title{
ÍNDICE DE QUALIDADE EM UM LATOSSOLO AMARELO COESO CULTIVADO COM CITROS ${ }^{1}$
}

\author{
JOSÉ FERNANDES DE MELO FILHO², LÍCIA LEMOS DE CARVALHO 3 , \\ DREID DE CERQUEIRA SILVEIRA ${ }^{4}$, JOSÉ AUGUSTO AMORIM SILVA DO SACRAMENTO ${ }^{5}$, \\ EDVANA CÁSSIA PINTO SILVEIRA ${ }^{6}$
}

RESUMO - O Brasil é o maior produtor mundial de citros e exportador de suco concentrado de laranja. Na região Nordeste, a citricultura tem grande importância social, onde é cultivada predominantemente por produtores com áreas menores do que 10 hectares, em solos como caráter coeso. Na Bahia, a citricultura é explorada sobre diversas condições tecnológicas, e especialmente nos Tabuleiros Costeiros os pomares têm baixa longevidade e produtividade em função das limitações dos solos coesos à produção agrícola. Este trabalho tem como objetivo determinar a qualidade física e química de um Latossolo Amarelo Coeso cultivado com citros. O estudo foi realizado na região do Recôncavo Baiano, em um pomar comercial de laranja "Pera" (Citrus sinensis L. Osb.) sob porta-enxerto de limão-cravo (Citrus limonia L. Osb.). Para a determinação do índice de qualidade do solo (IQS), utilizou-se o método proposto por Karlen \& Stott (1994), e amostras foram coletadas em duas camadas: 0,00 - 0,20 m e 0,20 - 0,40 m. Os resultados mostraram que o Latossolo Amarelo Coeso sob manejo tradicional para a cultura de citros apresentou índice de qualidade regular, com limitações determinadas pela elevada resistência do solo à penetração, baixa permeabilidade à água e baixo teor de matéria orgânica, o que resulta em limitações para permitir o crescimento e o aprofundamento do sistema radicular e prover o fornecimento e a disponibilidade de água para as plantas cítricas.

Termos para indexação: Tabuleiros Costeiros, qualidade do solo, laranja.

\section{QUALITY INDEX IN A COHESIVE YELLOW LATOSOL CULTIVATED WITH CITRUS}

\begin{abstract}
Brazil is the greatest citrus producer and exporter of concentrated orange juice in the world. In the Northeast, the citriculture has a large social importance for farming family, which is predominantly cultivated for small farmers. In Bahia state the citriculture has been exploited under several technology conditions and especially in the Costal table lands soil the orchards have low longevity and productivity based on the cohesive soil limitations for rural production. The purpose of this paper was to characterize the physical and chemical qualities of the cohesive yellow latosol with citrus crop in a conventional tillage system of the Coastal Table Lands in Bahia. The study was developed at the Recôncavo Baiano region, Brazil, from 2006 through 2007, where the orchards are usually cultivated with "Pera" sweet orange (Citrus sinensis L. Osb.) grafted on 'Rangpur' lime (Citrus limonia L. Osb.). It was used the soil quality index model proposed by Karlen $\&$ Stott (1994) and the soil samples were taken from the $0.00-0.20 \mathrm{~m}$ and $0.20-0.40 \mathrm{~m}$ layers. The results showed that under conventional tillage systems the citrus presented chemical and physical limitations for the root growth in depth, presenting a medium soil quality index, with limitations in $\mathrm{RP}_{100 \mathrm{kPa}}$, permeability and organic matter. The soil presents difficulties to maintain water supply and availability for citrus crops during the long period of water shortage, what demands the use of agricultural practices with the purpose of minimizing these problems, such as the use of cover crop, production and addition of organic material to soil and subsoiling.
\end{abstract}

Index terms: Costal table lands soil, soil quality, orange.

\footnotetext{
${ }^{1}$ (Trabalho 221-08). Recebido em: 27-08-2008. Aceito para publicação em: 08-10-2009. Parte da Dissertação de Mestrado do segundo Autor, apresentada ao Programa de Pós-Graduação em Ciências Agrárias do CCAAB/UFRB.

${ }^{2}$ Engenheiro Agrônomo, Doutor em Ciência do Solo, Universidade Federal do Recôncavo da Bahia - UFRB/CCAAB. Cruz das Almas - Bahia. Bolsista MEC/PET. E-mail: jfmelo@ufrb.edu.br.

${ }^{3}$ Engenheira Agrônoma, Mestranda do Programa de Pós-Graduação em Ciências Agrárias do CCAAB/UFRB. Bolsista CAPES. Email: licialemos@yahoo.com.br.

${ }^{4}$ Engenheira Agrônoma, Mestranda do Programa de Pós-Graduação em Ciências Agrárias do CCAAB/UFRB. Bolsista CAPES. Email: dreidcalsinal@hotmail.com.

${ }_{5}^{5}$ Aluno de Agronomia do CCAAB/UFRB, Bolsista PIBIC/CNPq. E-mail: jassacramento@yahoo.com.br.

${ }^{6}$ Engenheira Agrônoma, Mestre em Ciências Agrárias. E-mail: edvanapinto@yahoo.com.br
} 


\section{INTRODUÇÃO}

O Brasil é o maior produtor mundial de citros e exportador de suco concentrado de laranja. No País existe, aproximadamente, um milhão de hectares cultivados com citros, nos quais são produzidos 18 milhões de toneladas de frutas (IBGE, 2006) e gerados 1,50 bilhão de dólares anuais só com a exportação dos seus derivados. A região Sudeste é a maior produtora do País e, somente no Estado de São Paulo, essa atividade gera mais de 500 mil empregos diretos e indiretos.

$\mathrm{Na}$ região Nordeste, a citricultura também tem grande importância social. Nesta região, o citro é cultivado predominantemente por produtores com áreas menores do que 10 hectares. Na Bahia e Sergipe, segundo e terceiro produtores nacionais, a cultura de citros ocupa uma área de 108.319 hectares, representando $11 \%$ da área cultivada com a cultura no Brasil (IBGE, 2006; Azevedo, 2007). Nestes Estados, a citricultura é explorada sob diversas condições tecnológicas, desde as mais avançadas, com adoção de técnicas adequadas de preparo do solo, seleção de mudas e porta-enxerto, tratos culturais, correção de solo, adubação, nutrição das plantas, controle fitossanitário e índices elevados de produtividade, até as mais tradicionais, em maior número, apenas para subsistência e com baixos índices de produtividade, sem preocupações com o preparo adequado do solo, seleção das mudas e porta-enxerto e com realização eventual de controle fitossanitário e adubações (Souza et al., 2006).

Nos Tabuleiros Costeiros na Bahia, os pomares de citros apresentam baixa longevidade, entre 10 a 12 anos de efetiva produção, produzem baixo número de frutos por planta e não respondem às práticas usuais de adubação e controle fitossanitário (Rezende, 2000; Rezende et al., 2002). Nesse ambiente, os solos apresentam baixa qualidade e sérias limitações, tanto químicas quanto físicas, à produção agrícola (Jacomine, 1996; Souza, 1996; Carvalho et al., 1999; Cintra et al., 1999; Cintra et al., 2000; Rezende, 2000; Libardi \& Melo Filho, 2001; Melo Filho et al., 2007). Para Rezende (2000), o sistema de preparo do solo com arado e grade, quase sempre realizado de forma inadequada, com o solo muito seco, contribui para agravar as limitações dos solos coesos. Corroborando esses resultados, Portela et al. (2001) observaram que, em pomares de citros no Recôncavo da Bahia, o uso de roçadeiras no inverno e grade no verão ou mesmo grade durante todo o ano para o cultivo do solo, contribuiu para aumentar a densidade do solo, a resistência do solo à penetração e reduzir a infiltração de água. Portanto, o trabalho tem como objetivo determinar o índice de qualidade do solo (IQS), de um Latossolo Amarelo Coeso cultivado com citros em sistema tradicional de uso e manejo.

\section{MATERIAL E MÉTODOS}

O estudo foi realizado na região do Recôncavo da Bahia, no período de 2006/2007, em um pomar de cultivo tradicional de citros instalado na Fazenda Oliveira, no município de Cruz das Almas - BA, cujas coordenadas geográficas são: $12^{\circ} 40^{\prime} 12^{\prime}$ S, $39^{\circ}$ 06' $07^{\prime \prime} \mathrm{W}$, em uma altitude de $220 \mathrm{~m}$, temperatura média anual de $24,50^{\circ} \mathrm{C}$, clima tropical subúmido a seco, pluviosidade média de $1.224 \mathrm{~mm}$ por ano, com variações entre 900 e $1.200 \mathrm{~mm}$, sendo os meses mais chuvosos de março a agosto, e os mais secos de setembro a fevereiro (SEI, 2008).

A área é cultivada há 18 anos com laranja "Pera" (Citrus sinensis L. Osb.) sob porta-enxerto de limão-"cravo" (Citrus limonia L. Osb.), no espaçamento de $5 \mathrm{~m}$ entre linhas e $3 \mathrm{~m}$ entre plantas, totalizando uma área produtiva de, aproximadamente, 6,50 hectares. Na implantação do pomar, utilizou-se uma aração seguida de uma gradagem, e o plantio das mudas foi feito em covas de $0,40 \mathrm{~m}$ x 0,40 $\mathrm{m} \mathrm{x}$ $0,40 \mathrm{~m}$, adubadas com 2 litros de esterco de gado. $\mathrm{O}$ cultivo do solo para o controle das ervas infestantes é feito com grade, cinco vezes ao ano, nas entrelinhas das plantas. A colheita, realizada duas vezes ao ano, nos meses de junho e dezembro, é manual com carregamento via trator circulando nas entrelinhas das plantas. As adubações de cobertura, quando são realizadas, ocorrem na projeção da copa, com formulação comercial do tipo N-P-K, na proporção de 10-10-20, aplicando-se 0,20 kg por planta, conforme análise de solo para fins de fertilidade feita a cada três anos.

O solo da área foi classificado como Latossolo Amarelo Coeso (Embrapa, 2006), cujas características texturais (Embrapa, 1997) nas camadas de 0,00 - 0,20 m e 0,20 - 0,40 m estão indicadas na Tabela 1. As amostras foram coletadas nos pontos médios das camadas, nas entrelinhas da cultura, onde se marcou um transecto de $48 \mathrm{~m}$, com pontos de coleta espaçados de $12 \mathrm{em} 12 \mathrm{~m}$, totalizando cinco pontos de amostragem, conforme Souza (2005). Em cada ponto e profundidade, foram coletadas uma amostra deformada para as análises químicas e 5 amostras indeformadas para as análises físicas. A coleta das amostras deformadas foi realizada com trado pedológico, tipo sonda; e as indeformadas, com extrator de solo tipo Uhland, em anéis com diâmetro de 5,10 $\mathrm{cm}$, altura de $5,20 \mathrm{~cm}$ e volume de $106,18 \mathrm{~cm}^{3}$. 
Foram avaliados os seguintes indicadores de qualidade do solo e seus respectivos métodos: porosidade total, macro e microporosidade, densidade do solo e retenção de água em mesa de tensão e em câmaras de pressão de Richards (Embrapa, 1997); condutividade hidráulica no solo saturado pelo método do permeâmetro de carga decrescente (Libardi, 2005); capacidade de troca catiônica, saturação por bases, saturação por alumínio e pH, (Embrapa, 1997), e matéria orgânica pelo método de titulação após oxirredução por via úmida, conhecido como "Walkley \& Black", conforme descrito em Raij \& Quaggio (1983).

A curva de retenção da água no solo foi elaborada conforme o método descrito em Embrapa (1997). Na mesa de tensão, descrita por Kiehl (1979), amostras indeformadas de solo foram saturadas por capilaridade e submetidas aos potenciais matriciais de 0; 0,001; 0,002; 0,004 e 0,006 MPa. Paralelamente, na câmara de Richards (1965), amostras deformadas de solo foram colocadas em anéis de borracha sobre uma placa porosa, saturadas, e em seguida submetidas a tensões de 0,$01 ; 0,033 ; 0,10 ; 0,50$ e 1,50 MPa. Após atingir o equilíbrio, essas amostras foram pesadas em balança de precisão e, em seguida, levadas à estufa a $105^{\circ} \mathrm{C}$, por $48 \mathrm{~h}$, e pesadas novamente para a determinação da umidade correspondente à tensão aplicada. O conjunto completo de pares de dados de umidade versus tensão resultante do procedimento descrito acima foi ajustado ao modelo de van Genuchten (1980) ou:

$$
\theta=\left(\theta_{\text {sat }}-\theta_{\text {res }}\right)\left[1+(\alpha \cdot h)^{n}\right]^{-m}+\theta_{\text {res }}
$$

sendo: $\theta$ - umidade do solo $\left(\mathrm{m}^{3} \mathrm{~m}^{3}\right) ; \theta_{\text {sat }}$ - umidade do solo na saturação $\left(\mathrm{m}^{3} \mathrm{~m}^{3}\right) ; \theta_{\text {res }}$ - umidade residual do solo $\left(\mathrm{m}^{3} \mathrm{~m}^{3}\right) ; \mathrm{h}$ - tensão da água no solo $(\mathrm{MPa})$; $\alpha, \mathrm{m}$ e $\mathrm{n}$ - parâmetros de ajuste, adimensionais. Com este modelo, determinou-se a umidade do solo a 100 $\mathrm{kPa}$, cujo valor foi utilizado na curva de resistência do solo à penetração possibilitou o cálculo da $\mathrm{RP}_{100 \mathrm{kPa}} \mathrm{O}$ ajuste à equação de van Genuchten (1980) resultou nos parâmetros registrados na Tabela 2 .

Para a determinação da resistência do solo à penetração, utilizou-se o penetrômetro de impacto (Stolf et al., 1983). Os dados experimentais de resistência do solo à penetração foram obtidos em $\mathrm{Kgf} \mathrm{cm}^{-2}$ e depois multiplicados pela constante 0,098 para transformação em MPa (Beutler et al., 2001). Objetivando padronizar as medidas de resistência do solo à penetração para uma mesma umidade, neste caso, retida na tensão de $100 \mathrm{kPa}$ (Tabela 2), medidas de resistência do solo à penetração (RP) e umidade gravimétrica $(\mathrm{Ug})$ foram coletadas durante 6 meses, abrangendo desde a época mais úmida até a mais seca, de maneira a obterem-se medidas para diversas umidades no solo, o que gerou 8 pares de dados, que foram plotados em um gráfico de RP $\mathrm{x}$ $\mathrm{Ug}$, e equações de regressão foram ajustadas conforme Busscher et al. (1997), sendo possível obter-se o valor da RP para o teor de umidade do solo correspondente à tensão de $100 \mathrm{kPa}$.

O modelo de avaliação do índice de qualidade do solo foi o proposto por Karlen \& Stott (1994). Trata-se de um sistema aditivo que usa uma série de funções principais do solo, às quais são atribuídos pesos e são integradas conforme a seguinte expressão:

$$
I Q S=\Sigma(q W i x w t)
$$

em que: IQS é o índice de qualidade do solo, $q W i$ é o valor calculado para cada função principal e wt é um peso numérico atribuído para as mesmas. As funções principais são escolhidas de acordo com o objetivo da avaliação e devem ser acompanhadas de indicadores físicos, químicos e biológicos, relacionados diretamente com sua medida. Os pesos numéricos (wt) são atribuídos às funções de acordo com o grau de importância da mesma para o funcionamento do solo, no desempenho da função para a qual o índice está sendo calculado. O somatório dos pesos de todas as funções principais deve resultar, no máximo, em 1,0 (um). Esse é o valor do IQS para um solo ideal em relação ao objetivo considerado. Quando o solo apresenta limitações, os valores do IQS são menores que 1,00 , sendo 0,50 o limite crítico entre a boa e a má qualidade.

Após atribuir os pesos relativos para as funções, devem-se identificar e priorizar os indicadores que influenciam cada uma, em diversos graus. Seguindo o mesmo procedimento usado para as funções principais, devem-se atribuir pesos numéricos para os indicadores. Da mesma forma, o somatório geral dos pesos dos indicadores em cada nível deve ser 1,00 (um). Os indicadores de qualidade apresentam-se em diversas unidades de medida, sendo necessário, então, um fator de normalização para que possam ser corretamente somados. O mecanismo utilizado e sugerido por Karlen \& Stott (1994) é uma função para padronização de escores que foi desenvolvida para sistemas de engenharia por Wymore, em 1993 (Glover et al., 2000), a qual tem a seguinte expressão:

$$
v=\frac{1}{1+((B-L) /(x-L))^{2 S(B+x-2 L)}}
$$

em que: $v$ é a pontuação padronizada; $B$ é o valor crítico do indicador, cujo escore padronizado deve ser sempre 0,50 , e que estabelece o limite entre 
a boa ou má qualidade do solo; $L$ é o valor inicial ou mais baixo que o indicador possa expressar, podendo receber o valor de $0 ; S$ é a inclinação da tangente à curva no ponto correspondente ao valor crítico do indicador, e $x$ é o valor da propriedade ou indicador, medido no campo.

Neste estudo, foram definidas três funções principais: crescimento radicular em profundidade (CRP); condução e armazenamento de água (CAA) e suprimento de nutrientes ( $\mathrm{SN}$ ). O cálculo do IQS foi feito da seguinte forma:

$$
q(F P)=I_{1} x W_{1}+\ldots+I_{n} x W_{n}
$$

em que: $q(F P)$ é a contribuição parcial de cada função principal para o valor global do IQS; $I_{n}$ representa os valores dos diferentes indicadores da função principal avaliada, e $W_{n}$ são os pesos relativos atribuídos a cada indicador e representam a importância do mesmo na composição do índice de qualidade do solo.

$\mathrm{Na}$ sequência, multiplicam-se os valores encontrados em cada função principal pelo seu respectivo ponderador e efetua-se a soma dos resultados, determinando-se, assim, o índice de qualidade do solo, IQS, conforme equação 5 .

$I Q S=(q C R P \times w C R P)+(q C A A \times w C A A)+(q S N \times w S N)(5)$

em que: $q C R P$ é o valor ponderado da função principal, crescimento do sistema radicular em profundidade, qCAA é a função da capacidade de condução e armazenamento de água; $q S N$ é a função do suprimento de nutrientes, e $w$ são os ponderadores associados a cada função principal. Os resultados foram enquadrados de acordo com a classificação proposta por Souza (2005), em que IQS $\leq 0,50=$ ruim; $0,50<\mathrm{IQS} \leq 0,70=$ regular; $0,70<\mathrm{IQS} \leq$ $1,00=$ ótima.

Para esta avaliação, utilizou-se a média dos valores do IQS obtidos para as duas profundidades amostradas, conforme recomendado por Silveira (2007). Os valores dos indicadores de qualidade foram submetidos à análise de variância, e as médias, comparadas pelo teste t de Student, a 5\% de significância, conforme delineamento inteiramente casualizado, em que camadas foram consideradas como fator de variação (2), e os pontos de amostragem, como repetições (5).

\section{RESULTADOS E DISCUSSÃO}

Os resultados observados estão registrados nas Tabelas 3; 4 e 5. Verifica-se que as camadas estudadas apresentaram textura predominantemente arenosa com leve redução do teor da areia e incremento de argila em profundidade (Tabela 1). Considerando-se os valores médios dos indicadores de qualidade química das duas camadas avaliadas (Tabela 3), observa-se que o solo apresenta acidez média a moderada (Alvarez, 1999), caráter eutrófico (Tomé Júnior, 1997) e baixa saturação por alumínio. Os valores da capacidade de troca de cátions e o teor de matéria orgânica são baixos (Bahia, 1989; Lepsch, 1991), o que indica baixa disponibilidade de cargas e elevado potencial de perdas de nutrientes por lixiviação.

Quanto aos indicadores de natureza física (Tabela 3), verifica-se que a densidade do solo apresenta valor próximo do limite crítico de $1,59 \mathrm{~kg} \mathrm{dm}^{-3}$ (Alvarez, 1999; Souza et al., 2003). Porém, a resistência do solo à penetração $\left(\mathrm{RP}_{100 \mathrm{kP}}\right)$ apresentou-se limitante, pois os valores estão muito acima do limite crítico de 2,00 MPa (Taylor et. al., 1966). Na camada mais superficial, $0,00-0,20 \mathrm{~m}$, o valor da $\mathrm{RP}_{100 \mathrm{kPa}}$, de 5,31 MPa, apresenta-se muito alto, é quase três vezes maior que o seu limite crítico e mais do dobro daquele verificado na camada de 0,20 - 0,40 m, cujo valor, 2,38 $\mathrm{MPa}$, indica a classe alta de resistência do solo à penetração (Arshad et al., 1996).

As curvas de resistência do solo à penetração para as duas camadas avaliadas estão apresentadas na Figura 1. O ajuste potencial (Busscher et al., 1997) para a relação umidade versus resistência do solo à penetração resultou na equações 6 para a camada de $0,00-0,20 \mathrm{~m}$ e na equação 7 para a camada de $0,20-$ $0,40 \mathrm{~m}$, com $\mathrm{R}^{2}$ de 0,985 e 0,929 , respectivamente.

$$
\begin{aligned}
& R P=0,0217 U g^{-2,18} \\
& R P=0,0055 U g^{-2,79}
\end{aligned}
$$

Em que: RP é a resistência do solo à penetração $(\mathrm{MPa})$ e Ug é a umidade gravimétrica do solo $\left(\mathrm{kg} \mathrm{kg}^{-1}\right)$. Os resultados mostram que a relação da resistência do solo à penetração com a umidade se apresenta de maneira similar entre as camadas, sendo muito próximas quando o solo está úmido e maior na camada de $0,20-0,40 \mathrm{~m}$ para a condição de solo mais seco. No entanto, deve-se destacar que, mesmo para conteúdos de água mais elevados, o solo apresenta valores de RP acima de 2,00 MPa, o qual pode alcançar até $23 \mathrm{MPa}$ durante o processo de secamento, como verificado na camada de 0,00 - 0,20 m, situação que resulta em sérias limitações físicas para o crescimento e elongação das raízes das plantas cítricas nesta área de produção. Esta condição tem sido a realidade para a cultura de citros nos Tabuleiros do Recôncavo da Bahia e 
confirma os resultados encontrados por Dias (2006) e apresentados por Rezende (2000) e Rezende et al. (2002), sendo esta, possivelmente, uma das causas para a baixa longevidade e produtividade dos citros nesta região agrícola.

Os valores da macroporosidade estão adequados (Carter, 2002) e facilitam o fluxo de gases e a aeração do solo, não se constituindo, assim, em limitação ao crescimento radicular e à distribuição de água no perfil (Reynolds et al., 2002; Silva, 2005). Em média, nas duas camadas, a porosidade total apresenta-se próxima e abaixo do limite inferior de $35 \%$, considerado por Kiehl (1979) para solos com textura semelhante ao deste estudo. Outra característica da porosidade está relacionada com a sua distribuição por tamanho. Na camada de 0,20-0,40 m, apesar do baixo percentual de porosidade total, a distribuição atende à relação de $2 / 3$ de macroporos e 1/3 de microporos (Kiehl, 1979); no entanto, para a profundidade de $0,00-0,20 \mathrm{~m}$, essa relação está completamente fora do padrão, tendo em vista que os percentuais de macro e microporos praticamente se equivalem (Tabela 4 ).

Os valores relativos à porosidade (Tabela 4) refletem a densidade do solo, que, por sua vez, apresenta valores ainda não totalmente influenciados pelo trânsito das máquinas para os tratos culturais, mas não explicam os valores encontrados para $\mathrm{K}_{0}$ (Tabela 3). Os resultados dos outros indicadores relativos ao armazenamento de água, às relações $\mathrm{UV}_{33 \mathrm{kPa}} / \mathrm{PT}$ e $\mathrm{AD} / \mathrm{PT}$, mostram diferenças significativas entre as camadas. $\mathrm{O}$ valor da relação $\mathrm{UV}_{33 \mathrm{kPa}} / \mathrm{PT}$ foi inferior ao limite crítico estabelecido para este indicador (Souza et al., 2003) e, portanto, limitante para a camada de $0,00-0,20 \mathrm{~m}$, enquanto na camada de $0,20-0,40$ m se observou o contrário (Tabela 3 ).

A condutividade hidráulica do solo saturado $\left(\mathrm{K}_{0}\right)$ foi estatisticamente diferente entre as camadas (Tabela 3), porém restritiva ao movimento de água em ambas. Apresentou-se na classe lenta, na camada de 0,00 - 0,20 m, passando a muito lenta em 0,20 0,40 m (Lepsch, 1991). Importante ressaltar que a condutividade hidráulica foi, dentre os indicadores da função da condução e armazenamento de água, o único que se apresentou com valores muito restritivos, o que contribuiu para o baixo valor do IQS dessa função. Tendo em vista seu efeito na redistribuição e importância para o armazenamento de água, este resultado indica a dificuldade do solo em prover o fornecimento de água para as plantas cítricas durante os longos períodos sem recarga, o que demanda a utilização de práticas agrícolas que possam melhorar essa característica da área, a exemplo das plantas de cobertura, leguminosas ou gramíneas, escarificação, incorporação de resíduos orgânicos ou mesmo subsolagem. Certamente, essas práticas resultariam em melhorias nos teores de matéria orgânica, permeabilidade do solo à água e redução da resistência do solo à penetração, principais indicadores limitantes desta área.

Os resultados para o índice global de qualidade (IQS) mostram que os valores das duas camadas foram próximos (Tabela 5), pouco acima de 0,5, e estão classificados como regular (Souza, 2005). São resultados semelhantes aos encontrados por Dias (2006), quando avaliou o sistema tradicional de uso e manejo do solo para a cultura de citros no ambiente dos Tabuleiros Costeiros.

Comparando-se individualmente as camadas estudadas, verificou-se que, em 0,00 - 0,2 m (Tabela 5), a melhor função foi a de suprimentos de nutrientes $(\mathrm{SN})$, cujo valor foi muito próximo do limite superior da faixa regular de IQS estabelecida por Souza (2005). Para esta função do solo (SN), todos os indicadores apresentaram-se em condições favoráveis, à exceção da matéria orgânica, cujos valores foram muito baixos e limitantes. Esse mesmo efeito do manejo do solo em citros, baseado no uso de grade ou capina nas entrelinhas para controle de plantas infestantes sobre a matéria orgânica, foi constatado por Cintra et al. (1983) e confirmado por estudos mais recentes de Neves et al. (2005), quando compararam cultivo de citros com mata nativa em relação ao estoque de carbono orgânico. Da mesma forma, Auler et al. (2008) concluíram que a utilização das plantas de cobertura resulta em significativas melhorias nos indicadores químicos de qualidade do solo cultivado com citros, especialmente em relação aos teores de carbono orgânico, pH, V\% e CTC. $\mathrm{Na}$ camada de 0,20 - 0,40 m, a melhor função foi a de condução e armazenamento de água (FCAA), enquanto a CRP e SN foram limitantes (Tabela 5), refletindo a influência dos elevados valores da $\mathrm{RP}_{100 \mathrm{kPa}}$, e do baixo teor de matéria orgânica, respectivamente . 
TABELA 1 - Composição textural para duas profundidades de um Latossolo Amarelo Coeso cultivado com citros no Recôncavo da Bahia.

\begin{tabular}{ccccc}
\hline $\begin{array}{c}\text { Prof. } \\
\mathrm{m}\end{array}$ & Areia & $\begin{array}{c}\text { Silte } \\
\mathrm{g} \mathrm{kg}^{-1}\end{array}$ & Argila & $\begin{array}{c}\text { Classe } \\
\text { Textural }\end{array}$ \\
\cline { 5 - 5 } $\mathbf{0 , 0 0 - \mathbf { 0 , 2 0 }}$ & 757,40 & 69,20 & 173,40 & Francoarenosa \\
$\mathbf{0 , 2 0 - \mathbf { 0 , 4 0 }}$ & 682,40 & 57,60 & 260,00 & Francoargilo arenosa \\
\hline
\end{tabular}

TABELA 2 - Valores dos parâmetros de ajuste ao modelo de van Genuchten (1980) e teor de umidade do solo a $100 \mathrm{kPa}$ para duas profundidades de um Latossolo Amarelo Coeso, cultivado com citros no Recôncavo da Bahia.

\begin{tabular}{cccccccc}
\hline $\begin{array}{c}\text { Prof. } \\
\mathrm{m}\end{array}$ & $\alpha$ & $\mathbf{n}$ & $\mathbf{m}$ & $\theta_{\text {sat }}$ & $\begin{array}{c}\theta_{\text {res }} \\
\mathrm{m}^{3} \mathrm{~m}^{-3}\end{array}$ & $\theta_{100 \mathrm{KPa}}$ & $\mathbf{R}^{2}$ \\
\cline { 1 - 1 } & & & & & & & \\
$\mathbf{0 , 0 0 - \mathbf { 0 , 2 0 }}$ & 0,079 & 1,766 & 0,434 & 0,292 & 0,108 & 0,113 & 0,954 \\
$\mathbf{0 , 2 0 - 0 , 4 0}$ & 0,057 & 1,087 & 0,080 & 0,236 & 0,157 & 0,176 & 0,983 \\
\hline
\end{tabular}

TABELA 3 - Indicadores de qualidade para um Latossolo Amarelo Coeso em duas profundidades sob sistema tradicional de uso e manejo com citros, no Recôncavo da Bahia.

\begin{tabular}{|c|c|c|c|c|c|c|c|c|c|c|c|c|}
\hline \multirow{2}{*}{ Prof. } & \multicolumn{4}{|c|}{ FSN } & \multicolumn{4}{|c|}{ FCRP } & \multicolumn{4}{|c|}{ FCAA } \\
\hline & $\mathbf{p H}$ & CTC & $\mathbf{V}$ & MO & $\mathbf{m}$ & Ds & ${ }^{*} \mathbf{R} \mathbf{P}_{100 \mathrm{kPa}}$ & MP & $\begin{array}{c}\mathbf{U V}_{\text {з3КРа }}{ }^{\prime} \\
\mathbf{P T}\end{array}$ & $\begin{array}{c}\text { AD/ } \\
\text { PT }\end{array}$ & $\mathbf{K}_{0}$ & MP \\
\hline $\mathrm{m}$ & - & $\mathrm{cmol}_{\mathrm{c}} \mathrm{dm}^{-3}$ & $\%$ & $\mathrm{~g} \mathrm{~kg}^{-1}$ & $\%$ & $\mathrm{~kg} \mathrm{dm}^{-3}$ & $\mathrm{MPa}$ & $\mathrm{m}^{3} \mathrm{~m}^{-3}$ & - & - & $\mathrm{cm} \mathrm{h}^{-1}$ & $\mathrm{~m}^{3} \mathrm{~m}^{-3}$ \\
\hline $0,00-0,20$ & $6,1 \mathrm{a}$ & $5,4 \mathrm{a}$ & $76,2 \mathrm{a}$ & $6,7 \mathrm{a}$ & $0,95 \mathrm{a}$ & $1,51 \mathrm{a}$ & 5,31 & $0,16 \mathrm{a}$ & $0,39 a$ & $0,15 \mathrm{a}$ & $10,83 a$ & $0,16 a$ \\
\hline $0,20-0,40$ & $5,4 \mathrm{~b}$ & $4,1 b$ & $52,4 \mathrm{~b}$ & $5,1 \mathrm{a}$ & $8,5 b$ & $1,53 \mathrm{a}$ & 2,38 & $0,11 \mathrm{a}$ & $0,62 b$ & $0,21 b$ & $2,29 b$ & $0,11 \mathrm{a}$ \\
\hline
\end{tabular}

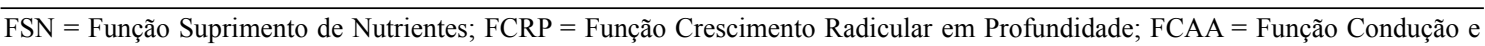
Armazenamento de Água. $\mathrm{RP} 100 \mathrm{kPa}=$ resistência do solo à penetração a $100 \mathrm{kPa}$ de tensão na umidade do solo; $\mathrm{MP}=$ macroporosidade do solo; $\mathrm{Ds}=$ densidade do solo; $\mathrm{m} \%$ = saturação por alumínio; $\mathrm{K} 0=$ condutividade hidráulica do solo saturado; $\mathrm{UV} 33 \mathrm{kPa} / \mathrm{PT}=$ relação umidade volumétrica retida a $33 \mathrm{kPa}$ /porosidade total; $\mathrm{AD} / \mathrm{PT}=$ relação água disponível/porosidade total; $\mathrm{CTC}=$ capacidade de troca catiônica; $\mathrm{V} \%$ = saturação por base, e $\mathrm{MO}=$ matéria orgânica. Médias seguidas pela mesma letra na coluna não diferem estatisticamente entre si, pelo teste t de Student, ao nível de $5 \%$ de probabilidade. *Dados sem comparação estatística. 
TABELA 4 - Distribuição da porosidade para um Latossolo Amarelo Coeso cultivado com citros, em sistema tradicional de uso e manejo, no Recôncavo da Bahia.

\begin{tabular}{cccc}
\hline Prof. & PT & MP & MI \\
& & & \\
\cline { 3 - 4 } & & & $\%$ \\
\cline { 3 - 4 } & & $16,52 \mathrm{a}$ & $19,93 \mathrm{a}$ \\
$\mathbf{0 , 0 0}-\mathbf{0 , 2 0}-\mathbf{0 , 4 0}$ & $36,46 \mathrm{a}$ & $11,34 \mathrm{a}$ & $21,13 \mathrm{a}$ \\
\hline
\end{tabular}

$\mathrm{PT}=$ Porosidade total; $\mathrm{MP}=$ Macroporosidade; $\quad \mathrm{MI}=$ Microporosidade. Médias seguidas pela mesma letra na coluna não diferem estatisticamente entre si, pelo teste $\mathrm{t}$ de Student, ao nível de 5\% de probabilidade.

TABELA 5- Índice de qualidade para um Latossolo Amarelo Coeso com citros em sistema tradicional de uso e manejo, no Recôncavo da Bahia.

\begin{tabular}{ccccc}
\hline $\begin{array}{c}\text { Prof. } \\
\mathbf{m}\end{array}$ & FCRP & FCAA & FSN & IQS \\
\hline $\mathbf{0 , 0 0 - \mathbf { 0 , 2 0 }}$ & 0,502 & 0,494 & 0,660 & 0,542 \\
$\mathbf{0 , 2 0 - 0 , 4 0}$ & 0,434 & 0,648 & 0,412 & 0,514 \\
\hline
\end{tabular}

FCRP = Função Crescimento Radicular em Profundidade; FCAA = Função Condução e Armazenamento de Água; SN = Função Suprimento de Nutrientes, e IQS = Índice de qualidade do solo.

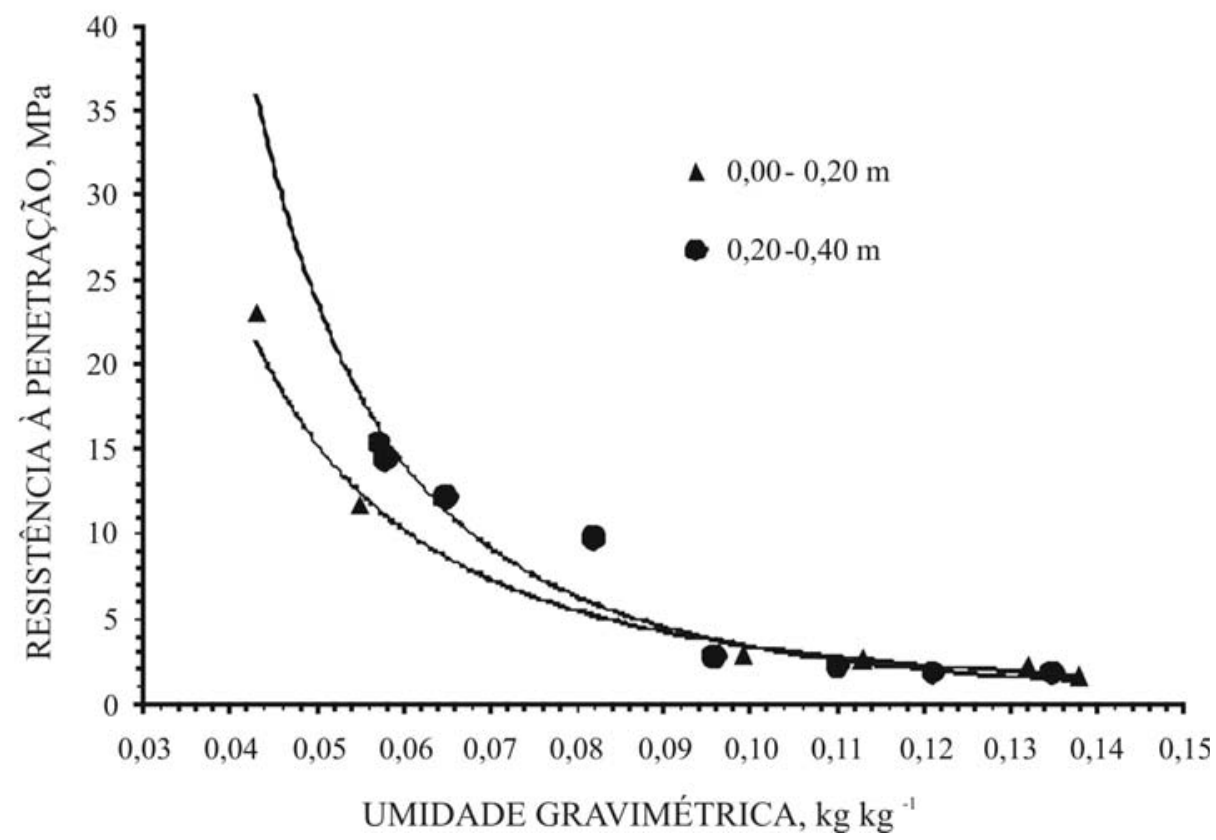

FIGURA 1 - Curva de resistência do solo à penetração para um Latossolo Amarelo Coeso sob cultivo com citros em sistema tradicional de uso e manejo. 


\section{CONCLUSÃO}

Verificou-se que o Latossolo Amarelo Coeso sob manejo tradicional para a cultura de citros apresentou índice de qualidade regular, com limitações determinadas pela elevada resistência à penetração, baixa permeabilidade à água e baixo teor de matéria orgânica, o que, provavelmente, resulta em restrições do solo para permitir o crescimento e o aprofundamento do sistema radicular e prover o fornecimento e a disponibilidade de água para as plantas cítricas.

\section{REFERÊNCIAS}

ALVAREZ, V. V.H.; NOVAIS, R.F.; BARROS, N.F.; CANTARUTTI, R.B.; LOPES, A.S. Interpretação dos resultados das análises de solos. In: RIBEIRO, A.C.; GUIMARÃES, P.T.G.; ALVARES, V.V.H. (Ed.). Recomendações para o uso de corretivos e fertilizantes em Minas Gerais $-\mathbf{5}^{\mathbf{a}}$ aproximação. Viçosa: Comissão de Fertilidade do Solo do Estado de Minas Gerais, 1999. p.25-32.

ARSHAD, M.A.; LOWERY, B.; GROSSMAN, B. Physical tests for monitoring soil quality. In: DORAN, J.W.; JONES, A.J. (Ed.). Methods for assessing soil quality. Madison: Soil Science Society of America, 1996. p.123 -141. (SSA Special Publication, 49).

AULER, P.A.M.; FIDALSKI, J.; PAVAN, M.A.; NEVES, C.S.V.J. Produção de laranja "pera" em sistemas de preparo de solo e manejo nas entrelinhas. Revista Brasileira de Ciência do Solo, Viçosa, v.32, n.1, p.363-374, 2008.

AZEVEDO. L. C. L. Produção Integrada de citros. Bahia, 2007. Disponível em: $<$ http://www.sistemasdeproducao.cnptia. embrapa.br.>. Acesso em : 20 nov. 008.

BAHIA. Comissão Estadual de Fertilidade do Solo. Manual de adubação e calagem para o Estado da Bahia. 2.ed. Salvador: CEPLAC/EMATERBA/ EMBRAPA/EPABA/NITROFÉRTIL, 1989. 173 p.

BEUTLER, A. N.; SILVA, M. L. N.; CURI, N.; FERREIRA, M. M.; CRUZ, J. C.; PERREIRA FILHO, I. A. Resistência à penetração e permeabilidade de Latossolo Vermelho distrófico típico sob sistemas de manejo na região dos cerrados. Revista Brasileira de Ciência do Solo, Viçosa, v.25, n.1. p.167-177, 2001 .
BUSSCHER, W.J.; BAUER, P.J.; CAMP, C.R.; SOJKA, R.E. Correction of cone index for soil water content differences in a coastal plain soil. Soil and Tillage Research, Amsterdam, v.43, n.3-4, p.205217, 1997.

CARTER, M. R. Quality: critical limits and standardization. In: LAL, R. (Ed.). Encyclopedia of soil science. New York: Marcel Dekker, 2002. p.1412-1421.

CARVALHO, J.E.B.; SOUZA, L. da S.; JORGE, L.A. de C.; RAMOS, W.F.; NETO, A. de O.C.; ARAÚJO, A.M. de A.; LOPES, L.C.; JESUS, M.S. de. Manejo de coberturas do solo e sua interferência no desenvolvimento do sistema radicular da laranja'Pera'. Revista Brasileira de Fruticultura, Jaboticabal, v.21, n.2, p.140 -145, 1999.

CINTRA, F.L.D.; LIBARDI, P.L.; SAAD, A.M. Balanço hídrico no solo para porta-enxertos de citros em ecossistema de Tabuleiro Costeiro. Revista Brasileira de Engenharia Agrícola e Ambiental, Campina Grande, v.4, n.1, p.3-28, 2000.

CINTRA, F.L.D.; LIBARDI, P.L.; JORGE, L.A.C. Distribuição do sistema radicular de porta-enxertos de citros em ecossistemas de Tabuleiro Costeiro. Revista Brasileira de Fruticultura, Jaboticabal, v.2, n.3, p.313-317, 1999.

CINTRA, F.L.D.; COELHO, Y.S.; CUNHA, SOBRINHO, A.P.; PASSOS, O.S. Caracterização física do solo submetido a práticas de manejo em pomar de laranja Baianinha. Pesquisa Agropecuária Brasileira, Brasília, v.18, n.2, p.173-179, 1983.

DIAS, R.C. dos S. Qualidade do solo e desenvolvimento radicular de citros em Latossolo Amarelo Coeso sob diferentes sistemas de manejo. 2006. 54 f. Dissertação (Mestrado em Ciências Agrárias) - Escola de Agronomia, Universidade Federal da Bahia, Salvador, 2006.

EMBRAPA. Centro Nacional de Pesquisa de Solo. Sistema brasileiro de classificação de solos. 2.ed. Rio de Janeiro, 2006. 306p.

EMBRAPA. Centro Nacional de Pesquisa de Solo. Manual de métodos de análise de solo. Rio de Janeiro, 1997. 212p. 
GLOVER, J.D.; REGANOLD, J.P. \& ANDREWS, P.K. Systematic method for rating soil quality of conventional, organic, and integrated apple orchards in Washington State. Agriculture, Ecossistems \& Environment, Amsterdan, v.80, n.1-2, p.29-45, 2000

IBGE. Instituto Brasileiro de Geografia e Estatística. Valor da produção agrícola municipal. Rio de Janeiro: IBGE/SIDRA, 2006. Disponível em: <http:// www.ibge.gov.br>. Acesso em: 11 mar. 2007.

JACOMINE, P.K.T. Distribuição geográfica, características e classificação dos solos coesos dos Tabuleiros Costeiros. In: REUNIÃO TÉCNICA SOBRE SOLOS COESOS DOS TABULEIROS COSTEIROS, 1., 1996, Cruz das Almas. Anais... Aracaju: Empresa Brasileira de Pesquisa Agropecuária, 1996. p.13-26.

KARLEN, D. L.; STOTT, D.E. A framework for evaluating physics and chemical indicators of soil quality. In: DORAN, J. W.; COLEMAN, D.C.; BEZDICEK, D.F.; STEWART, B.A. (Ed.). Defining soil quality for a sustainable environment, Madison: Soil Science Society of American, 1994. p. 53-71. (Special Publication, 35).

KIEHL, E. J. Manual de edafologia. São Paulo: Agronômica Ceres, 1979. 262p.

LEPSCH, I.F. Manual de levantamento utilitário do meio físico e classificação de terras no sistema de capacidade de uso. Campinas: Sociedade Brasileira de Ciência do Solo, 1991. 175p.

LIBARDI, P. L. Dinâmica da água no solo. 2. ed. Piracicaba: EDUSP, 2005. 329p.

LIBARDI, P.L.; MELO FILHO, J. F. Influência dos horizontes coesos na dinâmica da água no solo. In: WORKSHOP COESÃO EM SOLOS DOS TABULEIROS COSTEIROS, 2001, Aracaju. Anais... Aracaju: Embrapa Tabuleiros Costeiros, 2001. p.193-208.

MELO FILHO, J. F. de; SOUZA, A. L. V.; SOUZA, L. da S. Determinação do índice de qualidade subsuperficial em um Latossolo Amarelo Coeso dos Tabuleiros Costeiros sob floresta natural. Revista Brasileira de Ciência do Solo, Viçosa, v.31, n.6, p.1599-1608, 2007.
NEVES, C.S.V.J.; FELLER, C.; LARRÉ-LARROUY, M.C. Matéria orgânica das frações de um Latossolo Vermelho distroférrico sob diferentes sistemas de uso e manejo. Semina: Ciências Agrárias, Londrina, v.26, n.1, p.17-24, 2005.

PORTELA, J.C.; LIBARDI, P.L.; VAN LIER, Q. de J. Retenção da água em solo sob diferentes usos no ecossistema Tabuleiro Costeiro. Revista Brasileira de Engenharia Agrícola e Ambiental, Campina Grande, v.5, n.1, p. 49-54, 2001.

RAIJ, B. V.; QUAGGIO, J. A. Métodos de análise de solo para fins de fertilidade. Campinas: Instituto Agronômico, 1983. 31p.

REYNOLDS, W.D. ; BOWMAN, B. T. ; C. F. DRURY ; C. S. TAN ; LU X. Indicators of good soil physical quality: density and storage parameters. Geoderma, Amsterdam, v.110, n.1-2, p.131-146, 2002.

REZENDE, J. O. Solos coesos dos Tabuleiros Costeiros: limitações agrícolas e manejo. Salvador. SEAGRI- SPA, 2000. 117p. (Série Estudos Agrícolas, 1)

REZENDE, J. O.; MAGALHÃES, A.F. de J.; SHIBATA, R.T.; ROCHA, E.S.; FERNANDES, J.C.; BRANDÃO, F.J.C. ; REZENDE, V.J.R.P. Citricultura nos solos dos Tabuleiros Costeiros: análise e sugestões. Salvador: SEAGRI - BA., 2002. 97p.

SEI. Superintendência de Estudos Econômicos e Sociais da Bahia. Disponível em: $<$ http://www.sei.ba.gov.br/side/frame tabela.wsp?tmp.volta $=$ sg $46 \&$ tmp.tabela $=$ t $106>$. Acesso em: 03 mar. 2008.

SILVA, M. A. S. da; MAFRA, A. L.; ALBUQUERQUE, J. A.; BAYER, C.; MIELNICZUK, J. Atributos físicos do solo relacionados ao armazenamento de água em um Argissolo Vermelho sob diferentes sistemas de preparo. Ciência Rural, Santa Maria, v.35, n.3, p.544-552, 2005.

SILVEIRA, E.C.P. Qualidade do solo em dois sistemas de uso e manejo no ambiente dos Tabuleiros Costeiros do Recôncavo Baiano. 2007. 89f. Dissertação (Mestrado em Ciências Agrárias) - Centro 
de Ciências Agrárias e Ambientais, Universidade Federal do Recôncavo da Bahia, Cruz das Almas, 2007.

SOUZA, L. da S. Uso e manejo dos solos coesos dos Tabuleiros Costeiros. In: REUNIÃO TÉCNICA SOBRE SOLOS COESOS DOS TABULEIROS COSTEIROS, 1996. Cruz das Almas. Anais... Aracaju: Embrapa-CPATC/Embrapa-CNPMF/EAUFBA/ IGUFBA, 1996. p.36-75.

SOUZA, L. da S.; SOUZA, L.D.; SOUZA, L.F. da S. Indicadores físicos e químicos de qualidade do solo sob o enfoque de produção vegetal: estudo de caso para citros em solos coesos de Tabuleiros Costeiros. In: CONGRESSO BRASILEIRO DE CIÊNCIA DO SOLO, 29. , 2003, Ribeirão Preto. Palestras... Ribeirão Preto: Agromidia, 2003. CD-ROM.

SOUZA, A. L. V. Avaliação da qualidade de um Latossolo Amarelo Coeso argissólico dos Tabuleiros Costeiros, sob floresta natural. 2005. 95f. Dissertação (Mestrado em Uso, Manejo e Conservação dos Recursos Naturais Solo e Água) - Escola de Agronomia, Universidade Federal da Bahia, Salvador, 2005.
SOUZA L.D.; RIBEIRO, L. da S.; SOUZA. L da S.; LEDO. C. A da S.; SOBRINHO. A.P. da C. Distribuição das raízes dos citros em função da profundidade da cova de plantio em Latossolo Amarelo dos Tabuleiros Costeiros 2006. Revista Brasileira de Fruticultura, Jaboticabal, v.28, n.1, p.87-91, 2006.

STOLF, R.; FERNANDES, J.; FURLANI NETO, V. L. Recomendação para uso do penetrômetro de impacto modelo IAA/Planalsucar - Stolf. Revista STAB: açúcar, álcool e subprodutos, Piracicaba, v.1, n.3, p.11, 1983.

TAYLOR, H.M.; ROBERSON, G.M.; PARKER JR., J.J. Soil strength-root penetration relations to medium to coarse-textured soil materials. Soil Science, New York, v.102, n.1, p.18-22, 1966.

TOMÉ JÚNIOR, J. B. Manual para interpretação de análise de solo. Guaiba: Agropecuária, 1997. $247 p$.

VAN GENUCHTEN, M. Th. A closed form equation for predicting hydraulic conductivity of unsaturated soils. Soil Science Society of American Journal, Madison, v.44, p.892-898, 1980. 